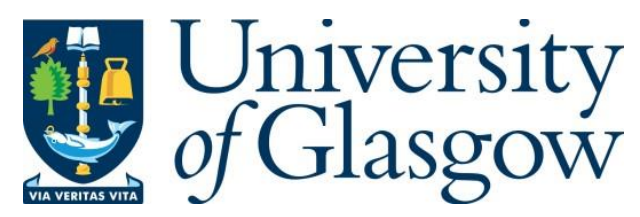

Marsh, J. H. and Hou, L. (2017) Integrated Gratings for Novel Photonic Integrated Circuits. In: Conference on Lasers and Electro-Optics/Pacific Rim, Sands Expo and Convention Centre, Singapore, 31 July - 4 Aug 2017, ISBN 9781509062904.

There may be differences between this version and the published version. You are advised to consult the publisher's version if you wish to cite from it.

http://eprints.gla.ac.uk/139416/

Deposited on: 7 April 2017

Enlighten - Research publications by members of the University of Glasgow http://eprints.gla.ac.uk 


\title{
Integrated Gratings for Novel Photonic Integrated Circuits
}

\author{
Invited Paper \\ John H. Marsh and Lianping Hou \\ School of Engineering \\ University of Glasgow \\ Glasgow, G12 8QQ, U.K. \\ john.marsh@glasgow.ac.uk
}

\begin{abstract}
Optical Bragg gratings are finding new applications in high frequency pulse generation and in multiple wavelength systems where great precision is required. Sampled Bragg gratings (SBGs) offer great design flexibility. Incorporating sampled grating reflectors in mode-locked semiconductor lasers allows stable pulse streams to be generated at $\mathrm{THz}$ frequencies. By using sampled Bragg gratings in distributed feedback laser arrays, the channel spacing can be defined in laser arrays with great precision. Furthermore, by introducing $\pi$-shifted sections into the SBGs, the grating coupling coefficient can be enhanced, opening new avenues for the design and low-cost manufacture of photonic integrated circuits.
\end{abstract}

Keywords-Semiconductor lasers; Bragg gratings; laser mode locking, quantum well lasers; laser arrays; distributed feedback lasers.

\section{INTRODUCTION}

For many years, optical Bragg gratings have been used in distributed feedback (DFB) and distributed Bragg reflector (DBR) lasers. The sampled grating distributed Bragg reflector (SGDBR), in particular, allows considerable design flexibility and is used in many designs of tunable DBR laser. In this paper, we will review the application of SGDBRs to high frequency mode-locked lasers and to DFB laser arrays. We will also demonstrate that phase shifted grating sections within the SGDBR can enhance the effective coupling coefficient, $\kappa$.

\section{SAMPLED BRAGG GRATINGS WITH PHASE SHIFTED SECTIONS}

In a conventional SGDBR (C-SGDBR), the grating is only present in defined sections of the waveguide (Fig. 1(b)). The effective coupling coefficient, $\kappa$, is necessarily reduced from that of a uniform grating (Fig. 1(a)) because much of the sampled grating period has no grating. To overcome this, designs of SBGs with phase shifted grating sections have been proposed that increase the effective $\kappa$. In these structures, the (not required) zeroth-order mode is suppressed while the index modulation experienced by the nonzeroth-order channels is enhanced, the \pm 1 -order channels being of particular interest [1].

This work was supported in part by the U.K. Engineering and Physical Sciences Research Council under Grant EP/E065112/1 and by the National Science Foundation of China under Grant 61320106013.
We will discuss the application of these principles to both mode-locked lasers and to high precision arrays of DFB lasers.

\section{HIGH FREQUENCY MODE-LOCKED LASER DIODES}

Generation of ultrafast and ultrashort optical pulses is of great interest for future large capacity optical communication networks. Mode-locked laser diodes (MLLDs) can produce short pulse widths ranging from picoseconds to subpicoseconds with small frequency chirp. In passive modelocking (ML), the longitudinal modes of a laser are phase locked by placing a saturable absorber (SA) adjacent to one facet. The laser then produces a series of pulses whose frequency $\left(F_{r}\right)$ is inverse of the cavity round trip time. Practical constraints on the cavity length limit the fundamental ML repetition frequency to $\sim 80$ to $170 \mathrm{GHz}$ for a MLLD with a typical cavity length between 0.5 and $1 \mathrm{~mm}$.

A robust solution for generating higher frequency pulse streams is to utilize harmonic ML (HML) at a multiple of the fundamental round-trip frequency of the device. This can be achieved by several methods, including colliding pulse ML and compound-cavity ML. However, fabrication tolerances make it difficult to obtain reliable ML at $F_{r}>\sim 200 \mathrm{GHz}$. We have therefore proposed techniques based on SGDBRs [2, 3]. The SGDBR provides strong frequency selectivity at the modelocked frequency while the 'soft walls' of the reflectors relax the tight fabrication tolerances required by other designs and ensure the long cavity can self-adjust to supporting an integral number of mode-locked periods. Using this approach, we have obtained pure ML operation at around $640 \mathrm{GHz}$ with nearly transform limited pulse characteristics. By tuning the bias parameters, operation at the second harmonic, with $F_{r}=1.28 \mathrm{THz}$, was also achieved [2].

To increase the coupling coefficient, as discussed in Section II we have applied a combination of $\pi$-phase shifted gratings, previously demonstrated in fiber lasers [1], with the C-SGDBR technique to $\mathrm{THz}$ repetition frequency MLLDs. Using a single electron beam lithography (EBL) step we have demonstrated a $620 \mathrm{GHz}$ side-wall SGDBR MLLDs with an increased effective $\kappa$ [4]. 
(a)

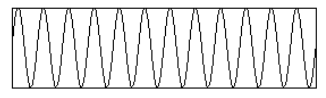

0 th order grating (b)

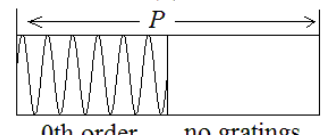

oth order no gratings (c)

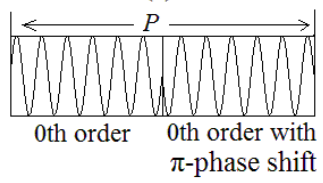

Fig. 1. Grating structures of (a) uniform $0^{\text {th }}$ order grating (b) C-SBG (c) PPS-SBG, $P$ is the sampling period

\section{DFB LASER ARRAYS}

DFB semiconductor laser arrays operating at precisely controlled wavelengths are important components for wavelength division multiplexing (WDM) networks. We have reported devices suitable for coarse WDM (CWDM) using conventional DFB lasers with uniform 0-order gratings (Fig.1 (a)) [5]. However, EBL cannot define the gratings with sufficient precision for dense WDM (DWDM) systems with small channel separation. For example, a resolution of about $0.125 \mathrm{~nm}$ would be required for an array with $100 \mathrm{GHz}$ spacing, which is beyond the typical resolution limit of $0.5 \mathrm{~nm}$ of EBL machines [6].

We have therefore realized precise wavelength control by applying the reconstruction-equivalent-chirp (REC) technique based on sampled Bragg gratings (SBGs) [6]. In contrast to conventional DBR lasers where lasing takes place at the 0order reflection peak from the grating, the REC approach uses either the +1 -order or -1 -order reflection. The wavelength of the refection is determined by the periodicity of the grating and by the dimensions of the sampling period $(P)$. The sampling period has dimensions of several $\mu \mathrm{m}$ or even $10 \mathrm{~s}$ of $\mu \mathrm{m}$, so it is relatively straightforward to define the wavelength separation between wavelength channels with great precision using conventional lithography.

Using REC, we have demonstrated a 4-channel PIC containing 4 REC-DFB lasers each with its own electroabsorption modulator, a $4 \times 1$ multi-mode interference coupler and a semiconductor optical amplifier [7]. Passive waveguide sections were bandgap widened using quantum well intermixing (QWI). Using a more conventional overgrowth fabrication process, we have also demonstrated the REC approach can deliver an exceptionally high yield to very large laser arrays; a 60-channel DFB laser array has been fabricated in which $88 \%$ of the laser wavelengths lay within a deviation of $\pm 0.20 \mathrm{~nm}[6]$

As discussed in Section II, the effective $\kappa$ of a sampled grating is substantially smaller than of a uniform grating. This compromises the single longitudinal mode (SLM) performance of DFB lasers. To overcome this, we have applied a combination of $\pi$-phase shifted SBGs (PPS-SBG) (Fig. 1(c)) with the REC technique to DFB diode lasers. Using a single EBL step we have demonstrated an increased effective coupling coefficient $\kappa$ and have fabricated an eight-channel laser array with a spacing of $100 \mathrm{GHz}(\sim 0.8 \mathrm{~nm} @ 1550 \mathrm{~nm})$ [8].

\section{CONCLUSION}

We have reviewed novel applications of lasers and PICs that make use of optical Bragg gratings. In particular, we have discussed high-frequency mode-locked lasers and high precision DFB arrays. All the devices and PICs can be fabricated using high-yield regrowth-free technology, in which gratings are surface etched into ridge waveguides and QWI is used to define passive waveguides. The PICs can therefore be manufactured at low-cost, making them eminently suited to price-sensitive applications such as optical access networks and data communications.

\section{ACKNOWLEDGMENT}

The authors would like to acknowledge the staff of the James Watt Nanofabrication Centre at the University of Glasgow for help in fabricating the devices.

\section{REFERENCES}

[1] J. Li, Y. Cheng, Z. Yin, L. Jia, X. Chen, S. Liu, S. Li and Y. Lu, "A multiexposure technology for sampled Bragg gratings and its applications in dual-wavelength lasing generation and OCDMA en/decoding," IEEE Photon. Technol. Lett., vol 21, pp. 1639-1641, 2009.

[2] L. Hou, M. Haji and J. H. Marsh, "Mode locking at Terahertz frequencies using a distributed Bragg reflector laser with a sampled grating," Opt. Lett. Vol 38, pp. 1113-1115, 2013.

[3] L. Hou, M. Haji and J. H. Marsh, "Mode-locking and frequency mixing at $\mathrm{THz}$ pulse repetition rates in a sampled-grating DBR mode-locked laser," Optics Express vol 22, pp. 21690-21700, 2014.

[4] L. Hou, S. Tang, and J. H. Marsh, "THz repetition frequency modelocked laser using novel sampled gratings," submitted to CLEO/Europe 2017.

[5] L. Hou, M. Haji, J. Akbar, J. H. Marsh and A. C. Bryce, "CWDM source based on AlGaInAs/InP monolithically integrated DFB laser array," Optics Lett., vol 36, pp. 4188-4190, 2011.

[6] Y. Shi, S. Li, X. Chen, L. Li, J. Li, T. Zhang, J. Zheng, Y. Zhang, S. Tang, L. Hou, J. H. Marsh and B. Qiu, "High channel count and high precision channel spacing multi-wavelength laser array for future PICs," Scientific Reports, vol 4, pp. 7377, 2014.

[7] L. Hou, J. Xu, I. Eddie, L. Han, H. Zhu and J. H. Marsh, (2016) "DWDM Source Based on Monolithic Side-Wall Sample Grating DFB Laser Array," 2016 Conference on Lasers and Electro-Optics (CLEO), San Jose, CA, 2016, (doi:10.1364/CLEO_SI.2016.SW4M.1)

[8] S. Tang, L. Hou, I. Eddie, X. Chen and J. H. Marsh, "Novel Sampled Grating Design for High Precision, Multiple Wavelength DFB Laser Arrays," Paper 84, European Conference on Integrated Optics (ECIO), Eindhoven, The Netherlands, 2017. 